\title{
A Quartz Crystal Microbalance Simulation to Examine the Effect of Ultraviolet Light Treatment on Characteristics of Polyethylene Surface
}

\author{
Keiko Gotoh ${ }^{1 *}$, Yumiko Tagawa ${ }^{2}$ and Izumi Tabata ${ }^{1}$ \\ ${ }^{1}$ Kyoto University of Education (1-Fukakusa-fujinomori-cho, Fushimi-ku, Kyoto 612-8522, JAPAN) \\ ${ }^{2}$ Kobe University of Fashion and Design (Meinan-cho, Akashi 673-0001, JAPAN)
}

\begin{abstract}
The effect of ultraviolet light irradiation on the characteristics of the polyethylene (PE) surface was investigated by the quartz crystal microbalance (QCM) technique. The PE film was prepared on the gold electrodes of the QCM by spin-coating from the solution and then was treated by the excimer UV lamp in ambient air. The changes in the hydrophilic properties, moisture adsorption, and water retention of the PE film due to the UV irradiation were determined from the frequency change of the QCM. To evaluate the detergency of the PE film, stearic acid as model oily soil was deposited onto the PE film formed on the QCM by the Langmuir-Blodgett (LB) technique, and was ultrasonically cleaned in aqueous detergent solutions containing ethanol or surfactant. The removal efficiency obtained from the frequency change of the QCM was found to increase considerably after the UV irradiation. From independently determined contact angles and the surface free energy components of the PE film, the free energy change resulting from the penetration of the detergent solution between stearic acid and PE in the zone of contact was calculated. Good relation was found between the removal efficiency and the free energy change, indicating that the increase in the detergency of the PE surface by UV irradiation was explained by surface energetics.
\end{abstract}

Key words: ultraviolet excimer light, quartz crystal microbalance, polyethylene, Langmuir-Blodgett films, wettability, detergency

\section{INTRODUCTION}

Synthetic polymers commonly are lacking in wettability, hygroscopicity, anti-static charge, prevention of soiling, and soil removal with washing and cleaning because of their hydrophobic property ${ }^{1,2)}$. Therefore, surface treatment by wet and dry processes has been utilized to enhance the hydrophilic nature of polymer surfaces ${ }^{3-9)}$. Recently, ultraviolet (UV) irradiation, one of the dry processes, has been applied to modify polymer surfaces ${ }^{10-13)}$. The UV irradiation can cause the photodissociation and ablation of the polymers, and therefore, change the chemical composition and the topography of their surface. In addition, the UV treatment has many advantages of the compact apparatus, exposure in ambient air, short required radiation time, and processing at low temperature.

In the present study, the effect of the UV treatment on surface characteristics of polyethylene was examined using a quartz crystal microbalance (QCM), sensitive mass measuring device ${ }^{14)}$. Polyethylene is most commonly used synthetic polymer and has extremely large hydrophobicity. The polyethylene film prepared on the QCM by a spin-coating technique was exposed to the $172 \mathrm{~nm}$ excimer lamp. Moisture adsorption and water retention on the PE film and the removal of oily soil from the PE film with cleaning in liquid were evaluated by the frequency change of the QCM. The experimental results were discussed on the basis of experimentally determined wettability and surface free energy components of the PE film.

\section{EXPERIMENTAL}

\subsection{Materials}

The AT-cut, 9MHz quartz crystal microbalance (QCM) was purchased from USI Systems Co. (Fukuoka, Japan), in which a gold electrode of $4.5 \mathrm{~mm}$ in diameter was deposit-

${ }^{*}$ Correspondence to: Keiko Gotoh, Kyoto University of Education, 1-Fukakusa-fujinomori-cho, Fushimi-ku, Kyoto 612-8522, JAPAN E-mail: Keiko@kyokyo-u.ac.jp Accepted May 26, 2008 (received for review April 24, 2008)

Journal of Oleo Science ISSN 1345-8957 print / ISSN 1347-3352 online

http://www.jstage.jst.go.jp/browse/jos/ 


\section{K. Gotoh, Y. Tagawa and I. Tabata}

ed on each side of a quartz plate. The QCM was cleaned twice with water, ethanol, and acetone prior to preparation of the PE film on its surface. The frequency of the QCM was measured with a frequency counter (HP 53131A/132A; Hewlett Packard, CA, USA). The mass of materials deposited on the electrodes of the QCM was calculated from the frequency change of the QCM using the Sauerbrey equation ${ }^{15,16)}$.

Polyethylene (PE) pellet (M.W.6500) commercially available (Scientific Polymer Products Inc., NY, USA) was dissolved in xylene $\left(10 \mathrm{~g} / \mathrm{dm}^{3}\right.$ ). Stearic acid (Wako Pure Chemical Industries, Ltd., Tokyo, Japan) was used as model soils, and was dissolved in benzene $\left(0.2 \mathrm{~g} / \mathrm{dm}^{3}\right)$ to prepare the spreading solution.

Other chemicals were extrapure grade and were used without further purification. The water was deionized, distilled using a Glass Still apparatus (Barnstead, Iowa, USA), and purified (resistivity of $18 \mathrm{M} \Omega \mathrm{cm}$ ) using a Easy Pure RF apparatus (Barnstead, Iowa, USA).

\subsection{Preparation of PE film on the QCM}

Immediately after dropping the PE solution on the gold electrodes, the QCM was spun at ca. 8000 rpm for 2-3 seconds and allowed to stand for 15 min for evaporation of the solvent. The procedure was repeated twice. The thickness of PE film formed on the gold electrodes was estimated to be $75 \pm 10 \mathrm{~nm}$ from the frequency change of the $\mathrm{QCM}$ due to the spin-coating. The mean roughness of the $\mathrm{PE}$ surface was determined to be $6.17 \mathrm{~nm}$ from the atomic force microscopic image ${ }^{17}$.

\subsection{UV irradiation}

The $172 \mathrm{~nm}$ UV irradiation to the PE film prepared on the QCM was carried out in ambient air using a $\mathrm{Xe}_{2}$ excimer vacuum UV apparatus (UER20-172, Ushio, Tokyo, Japan). The intensity of the UV excimer lamp at the upper window of the lamp house was determined to be 15.8 $\mathrm{mW} / \mathrm{cm}^{2}$ using a UV monitor system (UIT-150 and VUVS172, Ushio, Tokyo, Japan). The PE film surface was exposed to the UV light located $2 \mathrm{~mm}$ away from the window. In the previous study ${ }^{18,19)}$, the water contact angle on some polymer films decreased with UV irradiation time and showed saturation at 30-60 sec. Therefore, in the present study, the PE film was exposed to UV for $1 \mathrm{~min}$.

The mass loss of the PE film during the UV irradiation was determined to be $2.1 \pm 0.9 \%$ of total weight from the frequency change of the QCM. In general, the excimer lamp is not intense enough to cause ablation, although the excimer laser results in a photochemical reaction accompanied by surface ablation ${ }^{20,21}$. In the previous paper ${ }^{22}$, the PP and PET films were exposed to UV excimer lamp under the same condition in this study, and the resulted topographical change was much less than that reported in the case of the UV excimer laser treatment ${ }^{23}$. Therefore, it was considered that the mass loss observed was not due to clear ablation ${ }^{24)}$.

\subsection{Contact angle and surface free energy measurements}

The contact angles of the liquids on the PE and stearic acid surfaces and the surface free energies of liquids were measured by the sessile drop and the pendant drop methods, respectively, using a video contact angle system (VCA2500, AST Products, Inc., MA, USA). A 2-3 $\mu$ ldrop was placed on the PE film or Langmuir-Blodgett (LB) films of stearic acid (see 2.7). The stearic acid LB films as well as the PE film was prepared on the gold electrode of the QCM for experimental convenience, because the solid support did not affect significantly the contact angle. From the moment, the contact angle was determined every second from the drop profile stored in a computer. In the initial stage, the contact angle decreased linearly with time and the drop base diameter slightly increased. Therefore, the contact angle obtained by extrapolating the measured angles to time zero was obtained to be the advancing contact angle ${ }^{18)}$.

In the measurement of the surface free energy, the liquid was dispensed from the microsyringe to form a pendant drop, which was made as big as possible without letting it fall off the tip of the needle. The surface free energy was determined by pendant drop image analysis through videoimage digitization and numerical curve-fitting using the Laplace equation of capillarity ${ }^{25)}$.

\subsection{Evaluation of surface free energy of solid}

According to Lifshitz-van der Waals/acid-base (van Oss and Good) approach, the Young-Dupré equation for polar materials becomes ${ }^{26)}$ :

$\gamma_{2}(1+\cos \theta)=2\left[\left(\gamma_{1}^{\mathrm{LW}} \gamma_{2}^{\mathrm{LW}}\right)^{1 / 2}+\left(\gamma_{1}^{+} \gamma_{2}^{-}\right)^{1 / 2}+\left(\gamma_{1}^{-} \gamma_{2}^{+}\right)^{1 / 2}\right]$,

where $\theta$ is the contact angle of the liquid, $\gamma$ is the surface free energy, the subscripts 1 and 2 refer to the solid and the liquid, respectively, and the superscripts LW, +, and refer to the Lifshitz-van der Waals component, the Lewis acid parameter, and the Lewis base parameter, respectively. In the present study, the contact angles of three probe liquids, water, diiodomethane and ethylene glycol, were measured. The components of the surface free energies of the $\mathrm{PE}$ and stearic acid were calculated by the equation (1) using the measured advancing contact angles of three probe liquids and their published values of the surface free energy components ${ }^{27}$.

\subsection{Moisture adsorption and water retention}

The frequency of the QCM with the PE film can change due to the moisture adsorbed on its surface. Therefore, after standing for 20 min under two different humidity conditions, 30\%RH and 75\%RH, the frequency of the QCM was 
determined as $F_{1}$ and $F_{2}$, respectively. The mass change calculated from the difference between $F_{1}$ and $F_{2}$ was used as a parameter of moisture adsorption onto the PE film.

In the water retention measurement, the frequency of the QCM with the PE film, $\mathrm{F}_{3}$, was determined at $10 \mathrm{sec}$ after the $10 \mathrm{sec}$ immersion and pulling out of the water. The mass of water that wetted the PE film surface was estimated from the difference between $\mathrm{F}_{1}$ and $\mathrm{F}_{3}$.

\subsection{Soil removal experiments}

Prior to the soil removal experiment, stearic acid was deposited onto the PE film surface coated on the QCM by the LB deposition technique using a computer-controlled LB film deposition apparatus (FW-2, Lauda GmbH, LaudaKoenigshofen, Germany). The stearic acid monolayer on the water surface in the poly(tetrafluoroethylene) trough $\left(200 \times 600 \times 10 \mathrm{~mm}^{3}\right)$ was compressed continuously with a barrier speed of $8 \mathrm{~mm} / \mathrm{min}$ at $20 \pm 0.1^{\circ} \mathrm{C}$. The obtained surface pressure-area curve showed that a solid-condensed monolayer ${ }^{28)}$ was formed at a surface pressure of 30 $\mathrm{mN} / \mathrm{m}$. Therefore, at the constant pressure of $30 \mathrm{mN} / \mathrm{m}$, monolayers of stearic acid were transferred onto the $\mathrm{PE}$ film on the QCM by the vertical dipping method. The dipping velocity was $5 \mathrm{~mm} / \mathrm{min}$ and the dipping cycle was repeated ten times, corresponding to twenty layers deposition. The deposited mass of stearic acid onto the PE film surface, $M_{D}$, was calculated from the frequency change of the QCM owing to the deposition.

After aging 1-2 days, the QCM with soils was cleaned in $25 \mathrm{ml}$ aqueous $1 \mathrm{mmol} / \mathrm{dm}^{3}$ sodium chloride solutions containing ethanol or SDS ethanol by applying ultrasonic waves with the frequency of $42 \mathrm{kHz}$. The ultrasonic power was measured to be $20 \pm 5 \mathrm{mV}$ using a sound pressure meter (HUS-5, HONDA ELECTRONICS CO., Ltd). After $10 \mathrm{~min}$, the QCM was pulled out of the solution, and dried in air. In the case of the SDS solution, the solution uptake was displaced by pure water so that mass of SDS adsorbed on the QCM might not affect the experimental results. The mass of stearic acid removed from the PE surface, $M_{R}$, was calculated from the frequency change of the QCM due to the cleaning. The removal efficiency was expressed by $M_{R} / M_{D}$. In the previous paper ${ }^{17,29)}$, the removal efficiency of the fatty acid by ultrasonic washing showed saturation after $10 \mathrm{~min}$. Therefore, the removal efficiency in the present study was corresponding to the apparent equilibrium value.

All experiments were carried out in a room maintained at $25 \pm 1^{\circ} \mathrm{C}$.

\section{RESULTS AND DISCUSSION}

\subsection{Characteristics of PE film}

Figure 1 shows the water contact angle on the UV-treated $\mathrm{PE}$ film as a function of aging time. The increase in the angle, the hydrophobic recovery ${ }^{30,31)}$, was observed during aging in air. The recovery was remarkable within 3 days and almost complete after 1 week. Therefore, the UV-

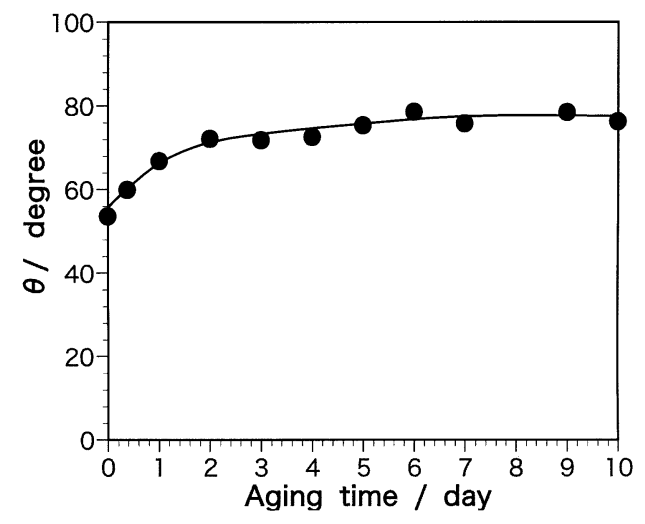

Fig. 1 The Contact Angle of Water, $\theta$, on the UV-treated PE Film as a Function of Ageing Time.

Table 1 Characterization of the PE Film Coated on the QCM before and after the UV Exposure.

\begin{tabular}{lccc}
\multicolumn{1}{c}{ Measurements } & Untreated PE & UV-treated PE \\
\hline Contact angle of water / degree & & 99.6 & 75.7 \\
Surface free energy / $\mathrm{mJm}^{-2}$ & $\gamma^{\mathrm{LW}}$ & 28.6 & 35.9 \\
& $\gamma^{+}$ & 0.1 & 0.7 \\
& $\gamma^{-}$ & 0.6 & 6.6 \\
& $\gamma^{\mathrm{T}}$ & 29.0 & 40.2 \\
Moisture adsorption / ng & & $67 \pm 15$ & $76 \pm 25$ \\
Water retention / ng & & $74 \pm 20$ & $8450 \pm 500$ \\
LB deposition / ng & & $1505 \pm 40$ & $325 \pm 100$ \\
\hline
\end{tabular}


exposed PE film was aged for 1 week prior to the experiments.

Table 1 shows the characteristics of the PE films before and after the UV irradiation. The water contact angle decreased, i.e. the wettability increased considerably by the irradiation. The acid and base parameters of the surface free energy also increased considerably by the UV irradiation. These results suggested that the UV irradiation caused surface functionalization. Although such change in surface characteristics was expected to affect the moisture adsorption onto the PE film surface, the difference in adsorbed moisture mass between untreated and UV-treated PE films was negligibly small. On the other hand, the adsorbed water mass onto the PE film was found to increase drastically after the UV irradiation. The deposited mass of the stearic acid LB films onto the untreated PE film was consistent with the calculated mass of twenty dry monolayers of stearic acid on two electrrodes (1502 ng). This indicated that the monolayers of stearic acid on water were completely transferred onto the $\mathrm{PE}$ surface as Y-type LB films ${ }^{28)}$. However, after the UV treatment of the PE film, the deposited mass onto its surface drastically decreased. This is a sign of unsatisfactory film deposition in which the change in molecular orientation and patchy deposition occurred because of poor adhesion of the hydrocarbon chains of stearic acid to the hydroplilic substrate surface.

\subsection{Effect of UV irradiation on soil removal}

The removal efficiency of stearic acid is presented in Fig. 2 as functions of ethanol and SDS concentrations. The removal efficiency increased by the additions of ethanol and SDS. At any concentrations of SDS and ethanol, the oil removal was enhanced considerably after the UV irradiation.

Such improvement of oily soil removal by the UV irradia-

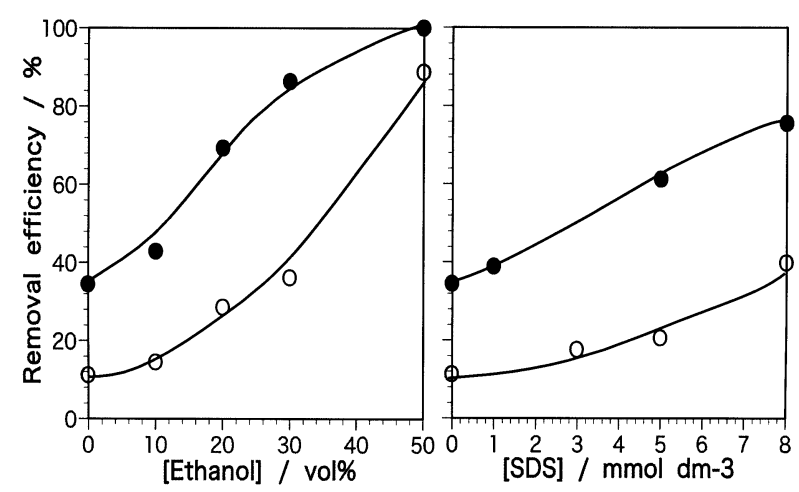

Fig. 2 The Removal Efficiency of Stearic Acid from Untreated (open circles) and UV-treated (closed circles) PE Films as Functions of Ethanol and SDS Concentrations. tion will be discussed on the basis of the wettability of the PE film. Figure 3 (open and close circles) shows the advancing contact angle of the detergent solution on the PE film as functions of ethanol and SDS concentrations. The angle decreased with increasing ethanol and SDS concentrations. At any concentration, the angle on the UVtreated PE film was smaller than that on the untreated PE film. From the experimental results in Figs. 2 and 3, the removal efficiency was plotted against the advancing contact angle (Fig. 4). The soil removal was found to increase almost linearly with decreasing contact angle. This suggested that the wettability enhancement in the PE film due to the UV irradiation promoted of the liquid penetration between stearic acid and $\mathrm{PE}$ in the contact zone leading to the increase of soil removal. Moreover, the results will be

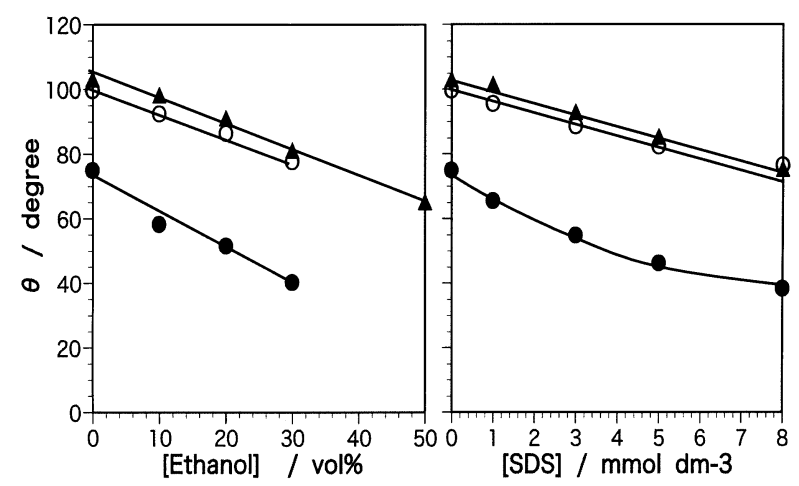

Fig. 3 The Advancing Contact Angles of the Detergent Solution on Untreated (open circles) and UV-treated (closed circles) PE Films and Stearic Acid (closed triangles) as Functions of Ethanol and SDS Concentrations.

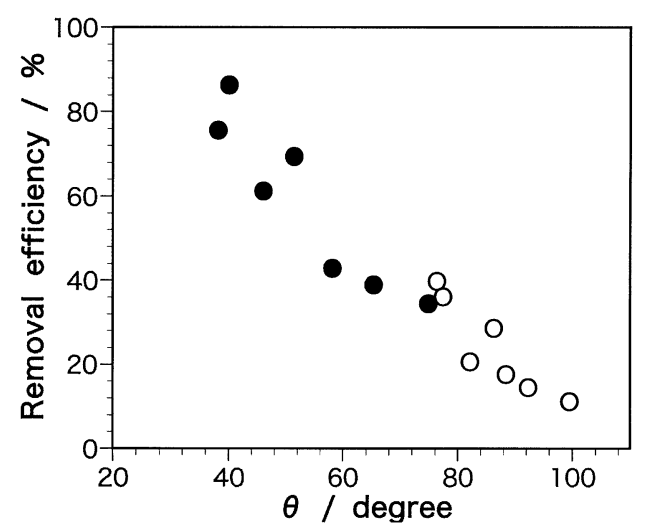

Fig. 4 Relation between the Removal Efficiency and the Advancing Contact Angle of the Detergent Solution on Untreated (open circles) and UV-treated (closed circles) PE Films. 
discussed in terms of the free energy change due to liquid penetration between stearic acid and the PE substrate.

The free energy change, $\Delta \mathrm{G}$, can be calculated as the displacement of the stearic acid/PE substrate interface by the stearic acid/liquid and PE /liquid interfaces as follows ${ }^{29,32}$ :

$\Delta \mathrm{G}=\gamma_{\mathrm{SL}}+\gamma_{\mathrm{PL}}-\gamma_{\mathrm{SP}}$

In this equation, $\gamma_{\mathrm{SL}}, \gamma_{\mathrm{PL}}$, and $\gamma_{\mathrm{SP}}$ are the interfacial free energies between stearic acid and the detergent solution, the PE film and the detergent solution, and stearic acid and the PE film, respectively. $\gamma_{\mathrm{SL}}$ and $\gamma_{\mathrm{PL}}$ are correlated with the contact angles by the Young equation:

$\gamma_{\mathrm{SL}}=\gamma_{\mathrm{S}}-\gamma_{\mathrm{L}} \cos \theta_{\mathrm{L} / \mathrm{S}}$

and

$\gamma_{\mathrm{PL}}=\gamma_{\mathrm{P}}-\gamma_{\mathrm{L}} \cos \theta_{\mathrm{L} / \mathrm{P}}$

where $\gamma_{\mathrm{S}}, \gamma_{\mathrm{P}}$, and $\gamma_{\mathrm{L}}$ are the surface free energies of stearic acid, the $\mathrm{PE}$ film, and the detergent solution, respectively. $\theta_{\mathrm{L} / \mathrm{S}}$ and $\theta_{\mathrm{L} / \mathrm{P}}$ are the contact angles of the detergent solution on stearic acid and the PE film, respectively. $\gamma_{\mathrm{SP}}$ is rewritten using the surface free energy components as follows ${ }^{33)}$ :

$\begin{aligned} \gamma_{\mathrm{SP}}= & {\left[\left(\gamma_{\mathrm{S}}^{\mathrm{LW}}\right)^{1 / 2}-\left(\gamma_{\mathrm{P}}^{\mathrm{LW}}\right)^{1 / 2}\right]^{2}+2\left[\left(\gamma_{\mathrm{S}}^{+} \gamma_{\mathrm{S}}^{-}\right)^{1 / 2}+\right.} \\ & \left.\left(\gamma_{\mathrm{P}}^{+} \gamma_{\mathrm{P}}^{-}\right)^{1 / 2}-\left(\gamma_{\mathrm{S}}^{+} \gamma_{\mathrm{P}}^{-}\right)^{1 / 2}-\left(\gamma_{\mathrm{P}}^{+} \gamma_{\mathrm{S}}^{-}\right)^{1 / 2}\right] .\end{aligned}$

The value of $\Delta \mathrm{G}$ was calculated by Equations 2-5 using the contact angles in Fig. 3, the surface free energies of the detergent solutions in Fig. 5, the surface free energy components of stearic acid $\left(\gamma_{\mathrm{S}}^{\mathrm{LW}}=22.9 \mathrm{~mJ} / \mathrm{m}^{2}, \gamma_{\mathrm{S}}{ }^{+}=0.1 \mathrm{~mJ} / \mathrm{m}^{2}\right.$, $\gamma_{\mathrm{s}}{ }^{-}=0.8 \mathrm{~mJ} / \mathrm{m}^{2}$ ) and the PE film (see Table 1).

Figure 6 demonstrates the relationship between the removal efficiency and $\Delta \mathrm{G}$. In all cases, the calculated values of $\Delta \mathrm{G}$ were positive, suggesting that stearic acid was removed by applying ultrasonic waves as the driving force of oily removal. The results obtained with the untreated and UV-treated PE films were plotted on almost the same line and the removal efficiency was found to increase with decreasing $\Delta \mathrm{G}$. This experimental finding shows that the penetration of the detergent solution between oily soil and the PE substrate in the zone of contact is a dominant factor of removal.

\section{CONCLUSION}

The quartz crystal microbalance coated with the PE film was utilized to obtain fundamental information on the UV treatment of the polymer surfaces. Water retention, and oily soil deposition and removal in liquid were successfully

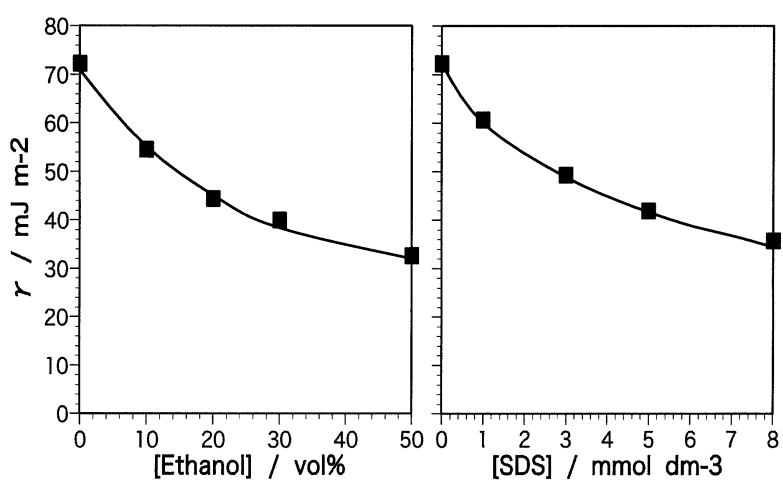

Fig. 5 The Total Surface Free Energy of the Detergent Solution as Functions of Ethanol and SDS Concentrations.

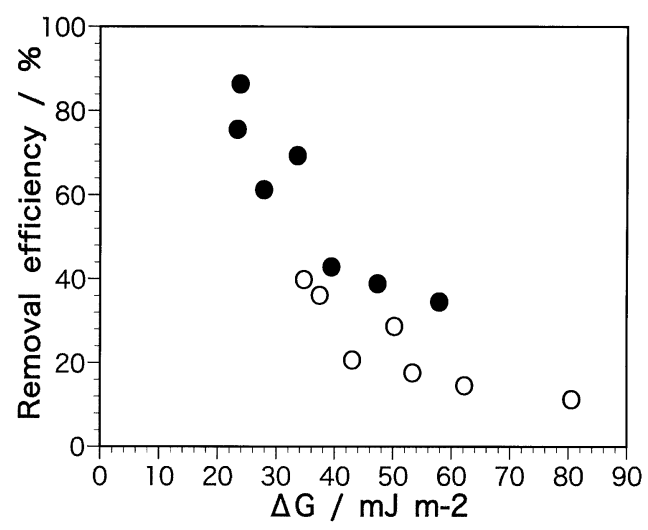

Fig. 6 Relation between the Removal Efficiency and the Free Energy Change due to the Penetration of the Detergent Solution between Stearic Acid and the PE Film in the Zone of Contact.

determined from the frequency change of the QCM. Together with the independently evaluated wettability and surface free energy, the UV irradiation was found to be effective for the modification of the PE surface.

\section{ACKNOWLEDGEMENT}

Gratitude is expressed to the Ministry of Education, Sports, Culture, Science and Technology, Japan for a Grant-in-Aid for Scientific Research to carry out this work. We also would like to thank Mr. N. Kishimoto of Kyoto University of Education for his experimental assistance. 


\section{Reference}

1. Mittal KL (ed.). Polymer Surface Modification: Relevance to Adhesion, VSP, Utrecht (1996).

2. Mittal KL (ed.). Polymer Surface Modification: Relevance to Adhesion Vol. 2, VSP, Utrecht (2000).

3. Sheng, E.; Sutherland, I.; Brewis, D.M.; Heath, R.J. Effect of the chromic acid etching on propylene polymer surfaces. J. Adhesion Sci. Technol. 9, 47-60 (1995).

4. Rozovskis, G.; Vinkevičius J.; Jačiauskienè J. Plasma surface modification of polyimide for improving adhesion to electroless copper coatings. J. Adhesion Sci. Technol. 10, 399-406 (1996).

5. Yun, H. K.; Cho, K.; Kim, J. K.; Park, C. E.; Sim, S. M.; Oh, S.Y.; Park, J.M. Effects of plasma treatment of polyimide on the adhesion strength of epoxy resin/polyimide joints. J. Adhesion Sci. Technol. 11, 95-104 (1997).

6. Toanles, Q.; Pireaux, J.J.; Caudano, R.; Leclere, P.; Lazzaroni, R. XPS/AFM study of the PET surface modified by oxygen and carbon dioxide plasmas: Al/PET adhesion. J. Adhesion Sci. Technol. 12, 999-1023 (1998).

7. Kim, E.-Y.; Kong, J.-S.; An, S.-K.; Kim, H.-D. Surface modification of polymers and improvement of the adhesion between evaporated copper metal film and a polymemr. 1. Chemical modification of PET. J. Adhesion Sci. Technol. 14, 1119-1130 (2000).

8. Źenkiewicz, M. Investigation on the oxidation of surface layers of polyolefins treated with corona discharge. J. Adhesion Sci. Technol. 15, 63-70 (2001).

9. Strobel, M.; Lyons, C.S. The Role of Low-molecularweight oxidized materials in the adhesion properties of corona-treated polypropylene film. J. Adhesion Sci. Technol. 17, 15-23 (2003).

10. Lazare, S.; Srinivasan, R. Surface properties of poly(ethylene terephthalate) films modified by farultraviolet radiation at $193 \mathrm{~nm}$ (laser) and $185 \mathrm{~nm}$ (low intensity). J. Phys. Chem. 90, 2124-2131 (1986).

11. Srinivasan, R.; Sutcliffe, E.; Braren, B. Analysis of the dynamics of the interaction of ultraviolet lasers with organic polymers. Laser Chem. 9, 147-154 (1988).

12. Walzak, M.J.; Flynn, S.; Foerch, R.; Hill, J.M.; Karbashewski, E.; Lin, A.; Strobel, M. UV and ozone treatment of polypropylene and poly(ethylene terephthalate). J. Adhesion Sci. Technol. 9 , 1229-1248 (1995).

13. Laurens, P.; Sadras, B.; Décobert, F.; Aréfi-Khonsari, F.; Amouroux. J. Laser-induced surface modifications of poly(ether ether Ketone): Influence of the excimer laser wavelength. J. Adhesion Sci. Technol. 13, 983-997 (1999).

14. Lu, C.S.; Lewis, O.J. Investigation of film-thickness determination by oscillating quartz resonators with large mass load. Appl. Phy. 43, 4385-4390 (1972).

15. Sauerbrey, G.Z. Verwendung von schwingquarzen zur wagung dunner schichten und zur mikrowagung, Zeitschrift fur Physik. 155, 206- 222 (1959).

16. Ariga, K.; Okahata, Y. In situ Characterization of Langmuir-Blodgett films during a transfer process. Evaluation of transfer ratio and water incorporation by using a quartz crystal microbalance. Langmuir 10, 3255-3259 (1994).

17. Gotoh, K.; Nakata, Y. Detergency of oily contaminants as assessed with a quartz crystal microbalance and Langmuir-Blodgett method. J. Jpn. Oil Chem. Soc. 53, 489-495 (2004).

18. Gotoh, K.; Nakata, Y.; Tagawa, M.; Tagawa, M. Wettability of ultraviolet excimer-exposed PE, PI and PTFE films determined by the contact angle measurements. Colloid and Surfaces A: Physicochemical and Engineering Aspects 224, 165-173 (2003).

19. Gotoh, K. Wettability and surface free energies of polymeric materials exposed to excimer ultraviolet light and particle deposition onto their surfaces in water, in Polymer Surface Modification: Relevance to Adhesion Vol. 3 (Mittal, K.L. ed.). VSP, Utrecht, pp. 125-137 (2004).

20. Watanabe, H.; Takata, T. Surface modification of poly(ethylene terephthalate) fiber by excimer light. $J$. Adhesion Sci. Technol. 8, 1425-1437 (1994).

21. Song, Q.; Netravali, A.N. Excimer laser surface modification of ultra-high-strength polyethylene fibers for enhanced adhesion with epoxy resins, Part 1 . Effect of laser operating parameters. J. Adhesion Sci. Technol. 12, 957-982 (1998).

22. Gotoh, K.; Kikuchi, S. Improvement of wettability and detergency of polymeric materials by excimer UV treatment. Colloid Polymer Sci. 283, 1356-1360 (2005).

23. Zeng, J.; Netravali, A.N. KrF excimer laser surface modification of ultrahigh molecular weight polyethylene fibers for improved adhesion to epoxy resins. Polymer Surface Modification: Relevance to Adhesion Vol. 3 (Mittal, K.L. ed.). VSP, Utrecht, pp. 159-182 (2004).

24. Dumont, T.; Lazare, S.; Lippert, T.; Wokaun, A. Changes in the etch rate of photosensitive polymers as a function of the pulse number. Appl. Phys. A79, 12711274 (2004).

25. MacLead, C.A.; Radke, C.J. A growing drop technique for measuring dynamic interfacial tension. J. Colloid Interface Sci. 160, 435- 448 (1993).

26. van Oss, C.J.; Good, R.J.; Chaudhury, M.K. Additive and nonadditive surface tension components and the interpretation of contact angles. Langmuir 4, 884-891 (1988).

27. Gotoh, K.; Tagawa, M.; Ohmae, N.; Kinoshita, H.; Tagawa, M. Surface characterization of atomic oxygen beam-exposed polyimide films using contact angle measurements. Colloid Polymer Sci. 279, 214-220 (2001).

28. Hann, R.A. Langmuir-Blodgett Films (Robert, G. ed.). 
Plenum Press, New York, pp.17-92 (1990).

29. Gotoh, K. The role of liquid penetration in detergency of Long-chain fatty acid. J. Surfactants Detergents 8, 305-310 (2005).

30. Everaert, E.P.; Van der Mei, H.C.; De Vries, J.; Busscher, H.J. Hydrophobic recovery of repeatedly plasmatreated silicone rubber. Part 1. Storage in air. J. Adhesion Sci. Technol. 9, 1263-1278 (1995).

31. Kim, B.K.; Kim, K.S.; Cho, K.; Park, C.E. Retardation of the surface rearrangement of $\mathrm{O}_{2}$ plasma-treated LDPE by a two-step temperature control. J. Adhesion Sci. Technol. 15, 1805-1816 (2001).

32. Lange, H. Physical Chemistry of Cleaning Action in Solvent properties of surfactant solutions (Shinoda, K, ed.). Marcel Dekker Inc., New York, p.156 (1967).

33. van Oss, C.J.; Giese R.F.; Costanzo, P.M. DLVO and Non-DLVO Interactions in Hectorite. Clay Clay Miner. 38, 151-159 (1990). 